\title{
Optimization Research of Injection-Production Mode in Fractured Buried Hill Reservoir
}

\author{
Yang Lina, Wang Xinran, Shi Changlin, Zhu Xiaolin, Gu Lihong
}

\begin{abstract}
Oilfield $\mathrm{Z}$ in Bohai bay is a fractured buried hill reservoir. Different from conventional sandstone reservoirs, the reservoir space is mainly divided into matrix and fracture system, which are quite different in reservoir physical properties and porous flow mechanism. It is easy to cause intra-layer interference of reservoir in the process of water flooding development. In order to improve the water flooding development effect in oilfield $Z$, and solve the problem of intra-layer interference of fracture and matrix system. Taking the actual characteristic parameters of oilfield $Z$ as reference, the development effect of oilfield under different injection-production modes is predicted by numerical simulation software. The results show that under the mode of weak injection and forcible production, the swept volume of water flooding in the model is the largest and the remaining oil distribution is the least. In order to verify the reliability of the numerical simulation results, three-dimensional physical simulation experiments was carried out, which simulate the development effect under different injection-production mode. The results show that the water-free recovery period is longer, the water cut rises slowly and the oil recovery is higher under the mode of weak injection and forcible production. The results of physical experiments are consistent with those of numerical simulation. The research results are applied to actual oilfield production. The injection-production mode in the original scheme has been adjusted. Compared with the original scheme design, the effect of oilfield development is improved, and the increment of daily oil production for average single well reaches $83 \mathrm{~m} 3$. It can provide a reference for the study of injection-production mode in fractured buried hill reservoirs of the same kind.
\end{abstract}

Index Terms - fractured buried hill reservoir; injection-production mode, numerical simulation, physical experiment.

\section{INTRODUCTION}

As a special offshore reservoir, fractured buried hill reservoirs generally take water flooding method to supplement formation energy. However, compared with conventional sandstone reservoirs, its storage medium is dual media of matrix and fracture. Because of complex fracture structure and irregular development, this kind of reservoir has strong heterogeneity, the matrix system also has a complex structure, which is mainly composed of a large number of micro-cracks and a small amount of dissolution porous,

Yang Lina, CNOOC EnerTech - Drilling \& Production Co. Ltd., Tianjin, China

Wang Xinran, Tianjin Branch of CNOOC Ltd., Tianjin, China.

Shi Changlin, CNOOC EnerTech - Drilling \& Production Co. Ltd., Tianjin, China.

Zhu Xiaolin, Tianjin Branch of CNOOC Ltd., Tianjin, China

Gu Lihong, CNOOC EnerTech - Drilling \& Production Co. Ltd., Tianjin, China. compared with fracture system, its permeability is lower ${ }^{[1-6]}$.Therefore, in the process of water flooding, it is very easy to cause injected water breakthrough along fractures, and the crude oil in the matrix system is different to be produced, resulting in lower water flooding efficiency of reservoirs ${ }^{[7-12]}$.The existing research on optimizing water injection modes are aimed at sandstone reservoirs with conventional pore structure mostly ${ }^{[13-18]}$, but less at fractured buried hills reservoirs. Current optimization of water injection mode for fractured reservoirs is mainly based on onshore oilfields ${ }^{[19-20]}$ As the first offshore buried hill fractured reservoir in China, There are great differences between offshore oilfields and onshore oilfields in recovery rate, injection-production well spacing, oilfield $\mathrm{Z}$ has no precedent for reference. In order to avoid premature breakthrough of injected water in the process of development, solve problems such as large decline of production in the initial stage of oilfield. It is urgent to optimize the development mode of water injection in reservoirs, so as to increase the utilization rate of injected water and achieve higher oil recovery.

\section{GENERAL INTRODUCTION OF OILFIELD}

Oilfield $\mathrm{Z}$ is located in the middle-north section of the western Liaoning low swell in Bohai Bay area. It is the largest metamorphic fractured reservoir in offshore oilfields in China [21-22]. The main formation is Archaean metamorphic buried hill, and the reservoir type is massive reservoir with weak bottom water and dual media. A lithology is dominated by light gray gneiss and its cataclastic rocks. The reservoir has the characteristics of fracture development and strong heterogeneity, the fracture linear density is 50 to 100 per meter, and the average width of the fracture is 0.5 to $1.0 \mathrm{~mm}$. The fracture is a high angle oblique joint with an inclination of 45 to 60 degrees. The thickness of the reservoir is 50 to $90 \mathrm{~m}$, and it has typical dual-porosity and single-permeability reservoir characteristics. The average total porosity of reservoir is $6.8 \%$ by logging interpretation, the average porosity is $1.1 \%$ and the average permeability is 280 to 900 $\mathrm{mD}$ in fracture system, the average porosity of is $5.7 \%$, and the average permeability is less than $1 \mathrm{mD}$ in matrix system. The oil reserves of matrix system account for $75 \%$ of the total oil geological reserves. The reservoir was put into production in 2009. Water flooding is adopted in the oilfield, the injection wells and production wells are all horizontal wells. The injection-production well spacing is 500 to 800 meter. Although the oilfield has high productivity in the early stage of production, it also faces the problem of rapid increase of water cut after water breakthrough. It is necessary to optimize injection-production mode in time. 


\section{RESEARCH ON NUMERICAL SIMULATION OF INJECTION-PRODUCTION MODE}

\section{A. Establishment of Numerical Simulation Model}

Referring to actual reservoir physical parameters, establishment of geological model by geological modeling software Petrel, and transformed it into a reservoir model that can be operated by the numerical simulation software Eclipse. The number of meshes is $60 \times 40 \times 30$, and the mesh step is 20 $\mathrm{m} \times 20 \mathrm{~m} \times 2 \mathrm{~m}$. According to the well pattern used in oilfield $\mathrm{Z}$, the injection wells are set at the bottom of the model, and the production wells are set at the top of the model, and the well types are all horizontal wells. The permeability of matrix system is $1 \mathrm{mD}$, the permeability of fracture system is 600 $\mathrm{mD}$. The total porosity is $5.5 \%$, the porosity of matrix system is $5.7 \%$, and that of fracture system is $1.1 \%$. The reserve ratio of matrix system and fracture system is $3: 1$. Relative permeability curve, high pressure physical parameters and rock stress sensitive parameters are taken from actual measured values of oilfield Z. Fracture permeability is taken as a standard parameter for historical matching.

\section{B. Design of Numerical Simulation Schemes}

According to the overall development strategy of the oilfield, and the law of buried hill fracture development, four different injection-production modes were designed and studied. As shown in Figure 1, there are four modes: forcible injection and forcible production, forcible injection and weak production, weak injection and forcible production and weak injection and weak production. Numbered as scheme 1 to scheme 4, respectively:

Scheme 1, forcible injection and forcible production mode, refers to that the injection wells and the production wells are located in the area of fracture development, that is, the permeability of fracture is much larger than that of matrix, and the ratio of fracture to matrix permeability is more than 80 .

Scheme 2, forcible injection and weak production mode, means that the injection wells are located in area with developed fractures and high permeability, while the production wells are located in areas with fractures less developed and low permeability. The area where the fracture is less developed refers to the area where the permeability ratio of fracture to matrix is less than 30 , while the area where the fracture is developed is the area where the permeability ratio of fracture to matrix is more than 80 .

Scheme 3, weak injection and forcible production mode, means that the injection wells are located in areas with less developed fractures and low permeability, while the production wells are located in areas with developed fractures and high permeability.

Scheme 4, weak injection and weak production mode, refers to that the injection wells and the production wells are located in areas with less fractures developed and lower permeability, and areas with developed fractures and higher permeability are used as porous flow channels between injection and production wells.

\section{Results of Numerical Simulation}

After the completion of water flooding under different injection- production modes, the swept area and remaining oil distribution of water flooding are shown in Figure 1.

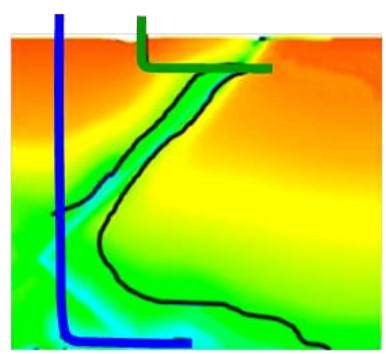

(a)

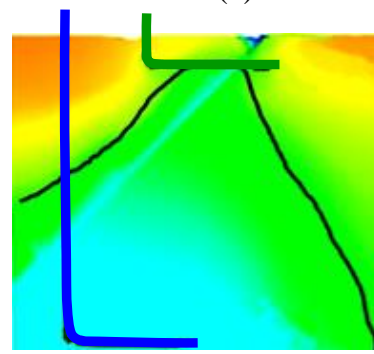

(c)
Production well Injection well

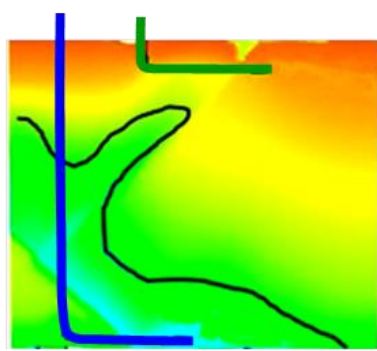

(b)

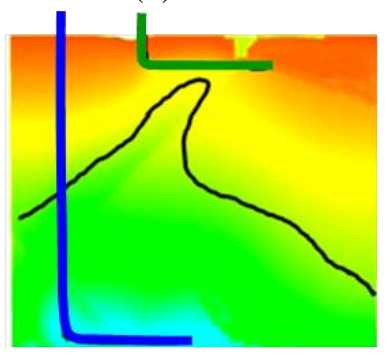

(d)
Figure 1 Remaining oil distribution of different injection-production mode scheme. (a) Forcible injection and

forcible production, (b) Forcible injection and weak production, (c) Weak injection and forcible production, (d) Weak injection and weak production.

Scheme 1 adopts forcible injection and forcible production mode. As shown in Figure 1 (a), injection wells are located in fracture developed areas; injected water porous flows rapidly along high permeability fractures. Injection-production pressure difference mainly concentrates in channeling channel, the fracture less developed areas and the matrix parts are few displaced. Only the bottom part of the model can be effectively displaced, and the swept area of the water drive front is the smallest. The fracture developed area is locally displaced in the production wells. The remaining oil in the upper part of the model is abundant, and the overall displacement effect of the model is poor.

Scheme 2 adopts forcible injection and weak production mode. As shown in Figure 1 (b), at the end of the injection well, the injected water flooding upward along the high permeability fracture. The middle and lower parts of the model have been flooded to some extent. Compared with scheme 1, the swept area of water flooding front increases obviously, and the remaining oil at the production well decreases accordingly. However, the production wells are located at the area of low fracture permeability, the production capacity is limited and the reservoir potential cannot be brought into full play. Its overall water flooding effect is better than scheme 1 , but its improvement effect is limited.

Scheme 3 adopts weak injection and forcible production mode. As shown in Figure 1 (c), fracture permeability at the end of injection wells is low, and the heterogeneity of surrounding reservoirs is weak. Injection water can flooding to the reservoir through the whole horizontal section, effectively inhibit the phenomenon of water flooding channeling. The displacement degree at the injection end of the model increases significantly, and the reaming oil saturation decreases greatly. The production wells are located in the area with high fracture permeability. When the injection water reaches the nearby of the production wells, water 
flooding channeling begins to occur. Because the whole production differential pressure at the bottom of the model has a large sweep range. The surrounding areas of main water channeling path can also be displaced to a certain extent. The swept area of water flooding front is the largest. At the same time, the production wells are located at high fracture permeability and have strong production capacity, which can give full play to the reservoir potential, the overall water flooding effect is the best.

Scheme 4 adopts weak injection and weak production mode. As shown in Figure 1 (d), weak reservoir heterogeneity at the location of the injection wells, that's increases the range of water flooding at the end of injection wells. At the same time, the weak heterogeneity of the location of the production wells, there is almost no phenomenon of water channeling in the model. The distribution range of injection-production pressure difference is wider and vertical displacement is more uniform. However, the low permeability of surroundings near the production wells, resulting in low productivity, the overall displacement effect is worse than that of scheme 3 .

The sweep volume coefficient of water flooding under different modes is calculated by area statistics method. As shown in Figure 2, the mode of forcible injection and forcible production is the easiest to form water channeling between fractures, and the sweep volume coefficient is the smallest, which is only $13.1 \%$. The sweep volume coefficient of water flooding increases only slightly in the mode of forcible injection and weak production, which is $25.7 \%$, because the productivity of production wells is small. The water flooding effect of weak injection and forcible production mode is the best, and the sweep volume coefficient is the largest, which is $59.9 \%$. The sweep volume coefficient of weak injection and weak production mode is $54.0 \%$, which lower than that of weak injection and forcible production mode in the same development time due to the low capacity of injection and production wells. 4 sets of contrast schemes show that when injection wells are located in areas with high fracture permeability, the development effect is poor when the strategy of forcible injection is adopted. When injection wells are located in areas with low fracture permeability, the development effect will be improved obviously by adopting the strategy of weak injection. It shows that injection mode can play a decisive role in the development of fractured reservoirs. Comparing the development effects of four injection-production modes, the weak injection forcible production mode is most suitable for the fractured buried hill reservoirs.

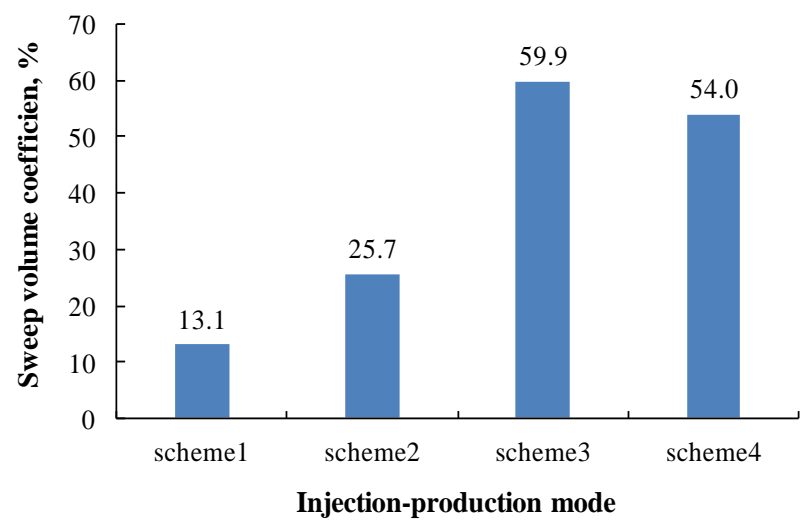

Figure 2 Sweep volume of different injection-production mode scheme

\section{PHYSICAL SimUlation EXPERIMENT OF INJECTION-PRODUCTION MODE}

\section{A. Establishment of Physical Simulation Experiment Model}

Considering that in actual fractured reservoirs, the nature and distribution of fractures are very complex. For the convenience of research and experiment, as shown in Figure 3, according to the classical Warren-Root model, the complex model is simplified to consist of a mutually vertical fracture system and rock blocks cut by the fracture system. According to the physical properties of reservoir rocks in actual oilfield $\mathrm{Z}$, the light brown reticulated granite with a large number of micro-fractures is selected as the experimental displacement medium. The average porosity and permeability of the rocks are $4 \%$ to $7 \%$ and $0.3 \mathrm{mD}$ to $1.0 \mathrm{mD}$, which are close to the actual situation of the oilfield. The rock is cut into cubic samples of two sizes, different fracture permeability models are simulated by cubic arrangement of different sizes.

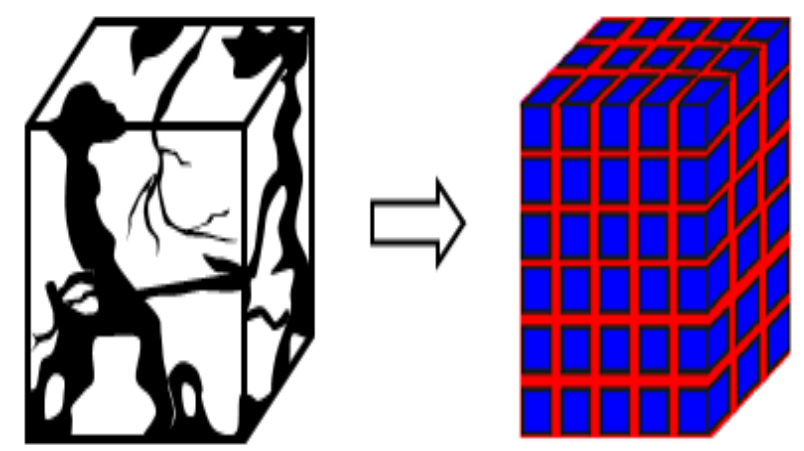

Figure 3 Diagrammatic sketch of Warren-Root model

\section{B. Scheme and process of physical simulation experiment}

Considering the investment cost and time cost of three-dimensional physical simulation experiment, only the two injection-production modes that mentioned above are compared.

Scheme 1, as shown in Figure 4(a), using small rock blocks with size of $5 \mathrm{~cm}$ and filter combination, a region with relatively developed fractures and high permeability is constructed. As shown in Figure 4(b), production well and injection well are located at the top and bottom of the model respectively, which form a forcible injection and forcible production mode.

Scheme 2, as shown in Figure 4(c), the bottom of the model is replaced by large rock blocks and yellow flakes with a size of $10 \mathrm{~cm}$ to construct relatively undeveloped fracture areas. As shown in Figure 4(d), production well and injection well are located at the top and bottom of the model respectively, which form a weak injection and forcible production mode.

The experimental process mainly includes: combination of blocks saturated with crude oil separately. Put it into a large-scale experimental device and add confining pressure to simulate formation pressure. Continue to saturate the crude oil to a stable state, and carry out water flooding experiments. 


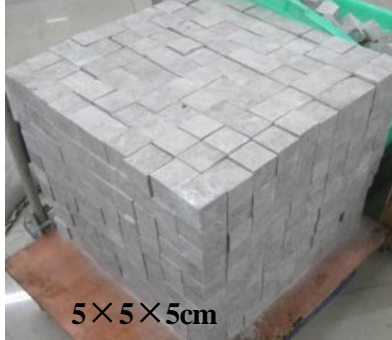

(a)

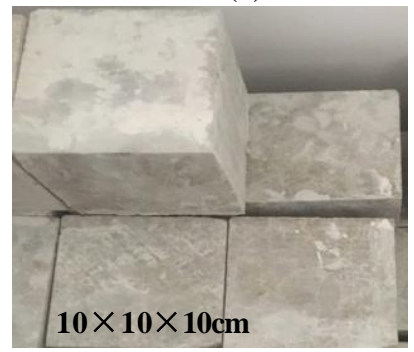

(c)

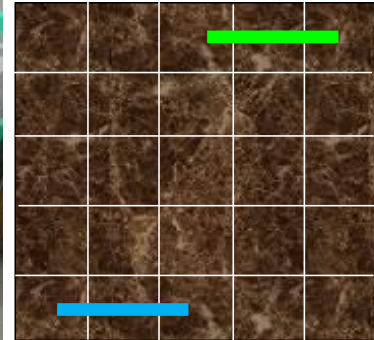

(b)

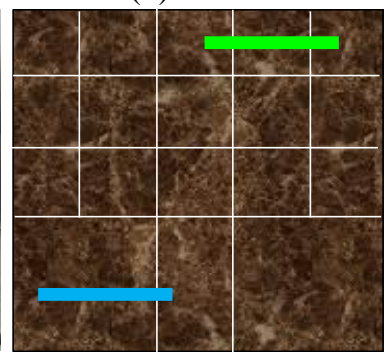

(d)
Production well

Figure 4 Injection-production mode scheme of

Three-dimensional physics simulation. (a) Small rock block, (b) Forcible injection and forcible production, (c) Big rock block, (d) Weak injection and forcible production.

\section{Results of Physical Simulation}

As shown in Figure 5, scheme 1 adopts forcible injection and forcible production mode. The model has a short water-free recovery period, and the water cut rises rapidly after water breakthrough. The oil recovery is only $17.4 \%$. Scheme 2 adopts weak injection and forcible production mode. The model has a long water-free recovery period, and the water cut increases slowly after water breakthrough. The oil recovery reach $21.0 \%$, the development performance of scheme 2 is better than that of scheme 1 . From the point of view of physical simulation, it is proved that the injection-production mode of "weak injection and strong production" in fractured reservoirs has better development effect. It can be used as the optimal injection-production mode for fractured reservoirs.

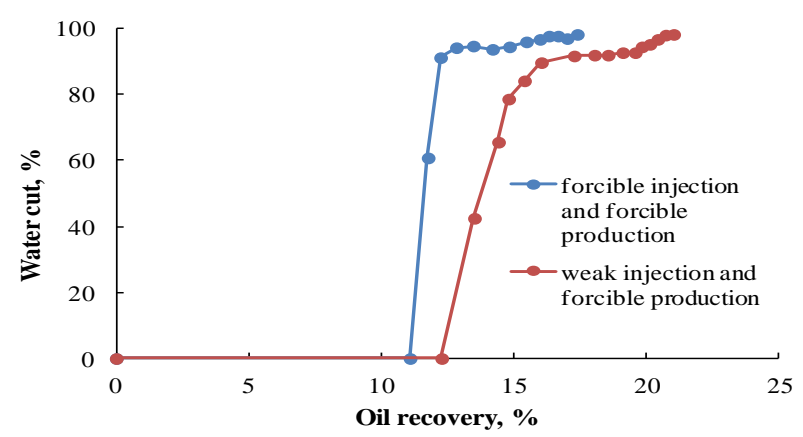

Figure 5 Production performance of different injection-production mode

\section{Field DEVELOPMENT PRACTICE}

As shown in Figure 6(a), both injection wells and production wells are located in the middle of fractured area of buried hill. According to this research result of injection-production mode optimization, the well pattern and well location in the original scheme are optimized. As shown in Figure 6(b), the optimized scheme designs that the production wells are located in the higher part of the structure, away from the oil-water interface as far as possible, and drilled into the fracture developed area. The injection wells are designed to be located in the fracture undeveloped area near the oil-water interface. At the same time, considering the reservoir of top-bottom staggered well pattern, and expanding the sweep scope of water flooding. In the optimization scheme, 2 injection wells and 5 production wells pattern is optimized to 3 injection wells and 5 production wells pattern, and to form weak injection and forcible production mode.

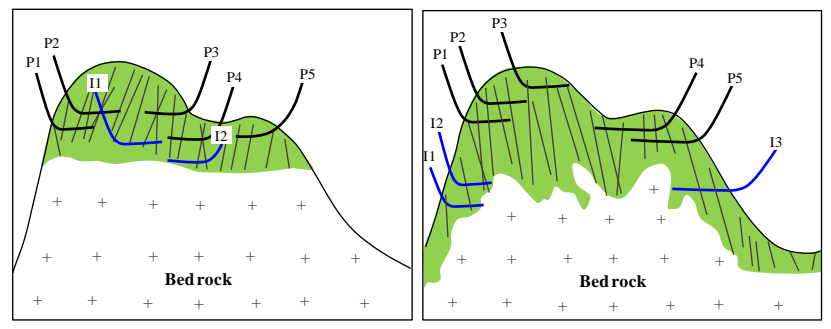

(a)

(b)

Figure 6 Injection-production mode and well location comparison of M block. (a) Original scheme, (b) Optimized scheme.

M block of oilfield $\mathrm{Z}$ was put into development in 2016. The comparison of daily oil production between the optimized scheme and the original scheme after development is shown in Figure 7. The actual daily oil production of 5 production wells in the initial stage exceeds the original scheme design. The average daily oil production of a single well exceeds $330 \mathrm{~m}^{3}$, compared with the original scheme, the average daily oil increase of a single well reaches $83 \mathrm{~m}^{3}$. The water-free recovery period also longer than that of the original scheme. The cumulative oil increase of the optimization scheme forecasted is $9.6 \times 10^{4} \mathrm{~m}^{3}$ more than that of the original scheme. The development of the new block has achieved better results, which shows that the results of this research can effectively guide the development and adjustment of fractured buried hill reservoirs.

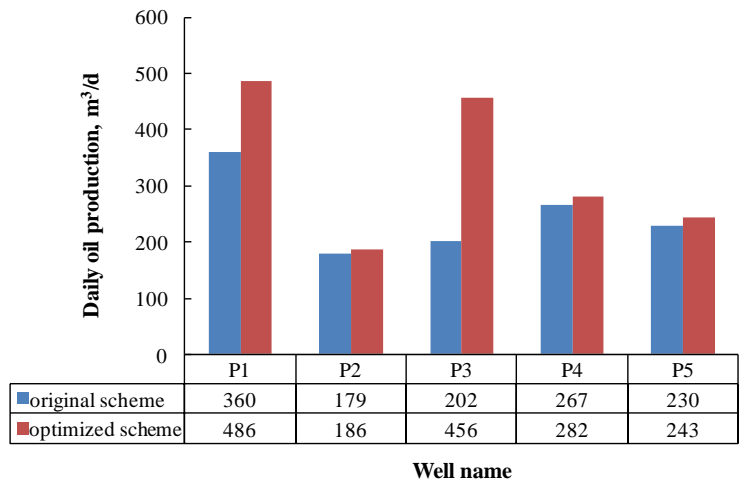

Figure 7 Daily oil production comparisons of original and optimized schemes

\section{CONCLUSIONS}

As shown in Figure 7(a), both injection wells and production wells are located in the middle of fractured area of buried hill. According to this research result of injection-production mode optimization, the well pattern and 
well location in the original scheme are optimized. As shown in Figure 7(b), the optimized scheme designs that the production wells are located in the higher part of the structure, away from the oil-water interface as far as possible, and drilled into the fracture developed area. The injection wells are designed to be located in the fracture undeveloped area near the oil-water interface. At the same time, considering the reservoir of top-bottom staggered well pattern, and expanding the sweep scope of water flooding. In the optimization scheme Fractured reservoirs in buried hills have complex reservoir development, In order to avoid water cut rising too fast and production decreasing sharply during the development of new block of oilfield Z, Referring to the actual characteristic parameters of the oilfield, numerical simulation and physical simulation experiments of fractured reservoirs are carried out. The injection-production mode of weak injection and forcible production is the optimal mode of oilfield development.

Field development practice shows that the injection-production mode of weak injection and forcible production can guide well location optimization of fractured reservoirs in buried hills, and play a positive role in improving water flooding effect of fractured reservoirs. The successful practice of this mode in oilfield $\mathrm{Z}$ has positive reference significance for the development of similar fractured buried hill reservoirs.

\section{REFERENCES}

[1] Wu, Y. S., Di, Y., Kang, Z. J. and Fakcharoenphol, P. A Multiple-Continuum Model for Simulating Single-Phase and Multiphase Flow in Naturally Fractured Vuggy Reservoirs[J]. Journal of Petroleum Science and Engineering, 2011, 78, 13-22.

[2] Guerreiro, V., Mazzoli, S., Iannace, A., Vitale, S., Carravetta, A. and Straus, C. A Permeability Model for Naturally Fractured Carbonate Reservoirs[J]. Marine and Petroleum Geology, 2013, 40, 115-134.

[3] Lorente, S. and Bejan, A. Heterogeneous Porous Media as Multi-Scale Structures form Maximum Flow Access[J]. Journal of Applied Physics, 2006, 100, 1149091-1149098.

[4] Liu, Q.Q. and Fan, H.G. An Approximate Method for Calculating the Equivalent Permeability Tensor of Seepage in Complex Fractures[J]. Mechanics in Engineering, 2012, 34, 16-20. (In Chinese).

[5] Warren, J.R. and Root, P.J. The Behaviour of Naturally Fractured Reservoirs[J]. Society of Petroleum Engineers, 1963, 228, 245-255.

[6] Cowie, P.A., Knipe, R.J. and Main, I.G. Scaling Laws for Fault and Fracture Populations-Analyses and Applications[J]. Journal of Structural Geology, 1996, 18, 135-383.

[7] Liu, H., Zhang, X., Lu, X., et al. Study on Flow in Fractured Porous Media Using Pore-Fracture Network Modeling[J]. Energies, 2017, 10, 1984.

[8] Koudina, N., Gonzalez, G.R. and Thovert, J.F. Permeability of Three-Dimensional Fracture Networks[J]. Physical Review E, 1998, 57, 4466-4479.

[9] Romero-Guzmán, E.T., Reyes-Gutiérrez, L.R., Klapp, J. and Hernández-Mendoza, H. Characterization of Natural Vuggy Fractured Porous Medium: Its Physicochemical Properties, Porosity and Permeability[J]. Journal of Minerals and Materials Characterization and Engineering, 2018, 6, 38-49.

[10] Agbogun, H.M.D., Al, T.A. and Hussein, E.M.A. Three Dimensional Imaging of Porosity and Tracer Concentration Distributions in a Dolostone Sample during Diffusion Experiments Using X-Ray Micro-CT[J]. Journal of Contaminant Hydrology, 2013, 145, 44-53.

[11] Giger, F.M. Analytic Two-Dimension Models of Water Cresting before Breakthrough for Horizontal Wells[J]. Reservoir Evaluation \& Engineering, 1989, 4, 409-416.

[12] Dou, H.E. Calculation of Critical Flow Rate for Horizontal Well in Bottom-Water Oil Reservoir[J]. Oil Drilling \& Production Technology, 1994, 21, 70-75.

[13] Muskat, M. and Wycokoff, R.D. An Approximate Theory of Water-Coning in Oil Production[J]. Transactions of the AIME, 1935, $114,144-163$.
[14] Xiong, X.D., Yang, J.J. and Liu, K. Determination of Water Injection Rate for Single Injection Well Employing Dividing Coefficient Approach[J]. Fault-Block Oil \& Gas Field, 2004, 11, 56-61.

[15] Suman, Y. K., Shirif, E., Ibrahim, H. and Ala-Ktiwi, A. Evaluation of Low Saline "Smart Water" Enhanced Oil Recovery in Light Oil Reservoirs[J], World Journal of Engineering and Technology, 2014, 2, 13-22.

[16] Abdulla, F., Hashem, H.S., Abdulraheem, A., Al-Naqi, M., Al-Qattan, A., John, H., Cunningham, P.R.P., Briggs, P.J. and Thawer, R. First EOR trial using low salinity water injection in the greater, Burgan field, Kuwait. SPE Middle East Oil \& Gas Show and Conference, Manama, 10-13 March 2013, Paper SPE-164341.

[17] Loahardjo, N., Xie, X., Yin, P. and Morrow, N.R. Low salinity waterflooding of a reservoir rock. International Symposium of the Society of Core Analysts[J], Calgary, 10-12 September 2007, Paper SCA2007-29.

[18] Jadhunandan, P. and Morrow, N. Effect of wettability on waterflooding recovery for crude oil/brine/rock systems[J]. Reservoir Engineering, 1995, 10, 40-46.

[19] Qin, S. G., Sun, Y. L., Jia. Z. Q. and Yang. D. G. Study of the Law about Water-Cut Variation for the Fractured Metamorphic Reservoir of Buried Hill with Bottom Water[J]. Energy and Power Engineering, 2009, 1, 44-49.

[20] Chen, S. S., Xu, K., Han, Y. Study on characteristics and development law of fractured basement reservoirs: A case study of fractured basement reservoirs in Damintun depression of Liaohe basin, 2003, SPE80541, 4, 1-9.

[21] Tong, K. J., Liu, H. Q., Zhang, Y. C., et al. Three-Dimensional Physical Modeling of Water flooding in Metamorphic Fractured Reservoirs[J]. Petroleum Exploration and Development, 2015, 42, 538-544.

[22] Luo, X. B., Li, Y. P., Ge, L. Z., Zhu, Z. Q., Zhang, L. L., Zheng, H. and Lv, Z. B. Research and Practice of Efficient Development Technology of Metamorphic Buried Hill Fractrue Reservoir[J]. China Offshore Oil and Gas, 2016, 28, 91-96. 